\title{
Autologous Bone Marrow Derived Mononuclear Cell Therapy for Vascular Dementia
}

\section{Sharma $A^{1}$, Badhe $\mathbf{P}^{1}$, Gokulchandran $\mathbf{N}^{1}$, Kulkarni $\mathbf{P}^{2 *}$, Sane $\mathrm{H}^{2}$, Lohia $\mathbf{M}^{3}$, Avhad $\mathbf{V}^{2}$ and Shetty $\mathrm{A}^{3}$}

${ }^{1}$ Department of Medical Services and Clinical research, NeuroGen Brain and Spine Institute, Surana Sethia Hospital and Research Centre, Suman Nagar, Sion-Trombay Road, Chembur, Mumbai, Maharastra, India

${ }^{2}$ Department of Research and Development, NeuroGen Brain and Spine Institute, Surana Sethia Hospital and Research Centre, Suman Nagar, Sion-Trombay Road, Chembur, Mumbai, Maharastra, India

${ }^{3}$ Department of NeuroRehablitaion, NeuroGen Brain and Spine Institute, Surana Sethia Hospital and Research Centre, Suman Nagar, Sion-Trombay Road, Chembur, Mumbai, Maharastra, India

\section{Abstract}

Background: Vascular dementia affects a broad spectrum of patients with various manifestations of cognitive decline, which are attributed to cerebral or cardiovascular disease. Laboratory studies have shown that transplanted bone marrow stem cells improve neurological diseases of the central nervous system by generating neural cells or myelin-producing oligodendroglial cells and enhancing neural plasticity. But till now, there has been lack of objective data in the form of investigational findings providing evidence for clinical improvements.

Method: We present a case of a 61 year old woman diagnosed with vascular dementia, who was administered autologous bone marrow derived mononuclear cells, intrathecally.

Result: Even after follow up of 2 years she showed sustained significant clinical improvements recorded by MMSE and FIM along with corroborating changes in PET CT scan of brain showing significantly improved metabolic activity.

Conclusion: Thus, demonstrating objective evidence showing benefits of neuroregeneration rehabilitation therapy in vascular dementia.

Keywords: Vascular; Dementia; Autologous; Bone marrow; Mononuclear cells; PET CT

\section{Introduction}

Vascular dementia (VaD) includes progressive loss of cognitive functions, evidence of cerebrovascular disease on brain imaging, focal signs of cerebrovascular disease and temporal relationship between stroke and dementia [1]. It results in an intellectual deficit which has a further significant impact on daily living, independence and relationships.

Interventions for vascular dementia are possible at a number of levels: primary prevention, secondary prevention, symptomatic treatments and disease modifying or curative approaches. There are various pharmacologic and nonpharmacologic therapies which have been tried as a treatment options. Currently, the medicines available are cholinesterase inhibitors, N-methyl-D-aspartate (NMDA) antagonists, and anti-depressants. Primary and secondary prevention is attempted by modification of vascular risk factors such as hypertension, hyperlipidemia and lifestyle. The drugs, cholinesterase inhibitors and memantine bring about only small cognitive improvements without global clinical outcomes and are also associated with adverse effects. Selective serotonin reuptake inhibitors and dihydropyridine calcium channel blockers may improve cognition only for a short-period [2]. Hence, at present there is no definitive treatment for $\mathrm{VaD}$. Recently, studies have been carried out to demonstrate the beneficial effects of bone marrow derived stem cells in dementia.

We describe a case of vascular dementia, which underwent autologous bone marrow derived mononuclear cell transplantation.

\section{Materials and Methods}

We present a right handed, 61 year old South Asian woman from India with a past medical history of hypertension and vascular dementia since 2002, showing history of in-coordination and imbalance while walking and a wide based gait. There was a noticeable deceleration in all her movements. Her orientation in time, place and person was also affected with apraxias. She noticed difficulty in swallowing, along with dysarthria and was emotionally labile. Gradually, she developed loss of short-term memory and decline in higher functions. Functionally, she required assistance for activities of daily living and supervision while walking and climbing stair. On the Functional Independence Measure (FIM), she scored 75/126. On psychological assessment using mini mental state examination (MMSE), she scored 10/30.

She was on neuroprotective medications such as Rivotril, Syndopa and Pramipexol. She underwent rehabilitation including physiotherapy, occupational therapy for over 4 years, but her symptoms continued to deteriorate.

On investigation, her CT scan showed diffuse cerebellar and cerebral atrophy while her MRI scan showed generalized cerebral and cerebellar volume loss. Small ischemic foci were also noticed in the bilateral frontal white matter (Figure 1). Angiography showed mild diffuse narrowing in M2 segment of right middle cerebral artery

*Corresponding author: Kulkarni P, Department of Research and Development NeuroGen Brain and Spine Institute, Surana Sethia Hospital and Research Centre Suman Nagar, Sion-Trombay Road, Chembur, Mumbai-400071, Maharastra India, Tel: 022-25281610/25283706; E-Mail: poojakul28@gmail.com

Received October 16, 2012; Accepted November 02, 2012; Published Novembe 04, 2012

Citation: Sharma A, Badhe P, Gokulchandran N, Kulkarni P, Sane H, et al. (2012) Autologous Bone Marrow Derived Mononuclear Cell Therapy for Vascular Dementia. J Stem Cell Res Ther 2:129. doi:10.4172/2157-7633.1000129

Copyright: () 2012 Sharma A, et al. This is an open-access article distributed unde the terms of the Creative Commons Attribution License, which permits unrestricted use, distribution, and reproduction in any medium, provided the original author and source are credited. 


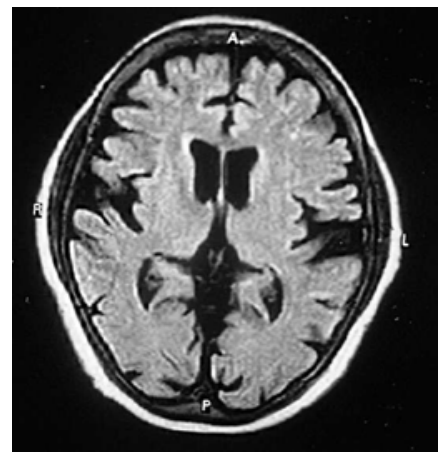

Figure 1: MRI Brain showing diffuse cerebellar and cerebral atrophy with small foci in frontal white matter

and mild tortuosity of neck vessels suggestive of atherosclerotic changes. Positron emission tomography (PET) scan showed global hypometabolism in the brain. Bilateral parietal lobe showed moderately reduced FDG uptake and bilateral frontal and temporal showed mild reduction. Left thalamus and right basal ganglia showed moderate reduction and both cerebellum showed mild reduction (Figure 2A).

Patient was selected as per the inclusion criterion based on paragraph 35 of the World Medical Association Declaration of Helsinki- Ethical Principles for Medical Research Involving Human Subjects [3]. The protocol had been reviewed and approved by the Institutional committee for Stem cell Research and Therapy (IC-SCRT) for our institution in Mumbai, India on 15 December 2008. Due to the history of dementia in the patient, her relative was informed about the procedure in details and a duly filled informed consent form was signed by the relative. Bone marrow $(100 \mathrm{ml})$ was aspirated from the iliac bone. Mononuclear cells (MNCs) obtained by density gradient separation, were free of pathogens. Viable count of the isolated MNCs was taken and was also checked for CD34+ markers by FACS analysis. She was administered $56 \times 10^{6} \mathrm{MNCs}$, which were dissolved in her own CSF, intrathecally. They consisted of $0.45 \%$ CD $34+$ cells. Two doses of MNCs were given at the interval of 3 months following the same protocol. The stem cell transplantation was also supplemented with physiotherapy, occupational therapy and speech therapy.

\section{Results}

Following autologous bone marrow derived mononuclear cell transplantation; the patient had no side effects and was evaluated at regular time intervals of 3 months, 6 months and 2 years.

On physical assessment, after 3 months, she showed improved fine motor activities. Cognitively, she was more alert and oriented, as compared to before the therapy. On neuropsychological assessment she showed improved memory, logical thinking, concentration, attention, reasoning and visuo-spatial skills such as perception, depth and mathematical calculations.

Her score on the MMSE had improved from 10/30 to 13/30. She now is independent in her daily functional activities such as eating, toilet activities along with improved dynamic trunk balance. Her coordination improved with ability to perform tandem walking with ease, which was not at all possible prior to the therapy.

On follow up after six months, functional improvements were further enhanced with respect to daily activities and cognition. At the end of 2 years, the progression of dementia appeared to have stalled which was otherwise rapidly progressing. On MMSE, her scores improved from $13 / 30$ to $16 / 30$ at 6 months follow up and finally to $20 / 30$ at the end of 2 years. Her FIM score improved from 75 to 80 after 2 years.

In addition, the PET scan repeated after 2 years, showed moderate reduced uptake of FDG only in the Wernicke's area, the insula, the lateral temporal and lobulus quadrilatere areas of the right hemisphere in the brain (non-dominant side). While the rest of the brain showed normal FDG uptake (Figure 2B).

\section{Discussion}

$\mathrm{VaD}$ is considered as the second most common cause of dementia in the elderly after Alzheimer's disease [4]. Between 1 to $4 \%$ of people over 65 years suffer from $\mathrm{VaD}$ [5].

It is assumed to be the outcome of lesions in the vascular distribution of the posterior or anterior circulation, which causes alterations in awareness, memory deficits, and abulia and may also produce disturbances in neuropsychological testing [6].

Inspite of being a common condition, there are no effective approved pharmacological treatments available. Large randomized studies have been carried out for the symptomatic treatment of probable and possible $\mathrm{VaD}$ which includes studies of the anticholinesterase donepezil in $\mathrm{VaD}$ [7], the NMDA receptor antagonist memantine and a study of the anticholinesterase galantamine $[8,9]$. Experimental studies have also been carried out to demonstrate the efficacy of stem cells for vascular dementia and other neurological disorders [10-12]. Studies have shown that the bone marrow-derived stem cells are mobilized from bone marrow and home into the injured brain [13].

Experiments in rats suggest that bone marrow derived mesenchymal stem cells (BMSCs) transplanted into the subventricular zone (SVZ) most likely function for a short term, by interacting with the host brain and stimulating recovery of impaired brain function [14]. BMSCs have been shown to secrete an array of neurotrophins, growth factors, and other supportive substances such as BDNF, NGF, VEGF and glial derived neurotrophic factor. This can help in improved survival of the neurons through facilitation of neuroprotective responses such as angiogenesis, axonal guidance and regeneration, which may further result in improved cognitive function of aging vascular dementia in rats [14]. CD34 antigen is present on immature hematopoietic precursor cells and all hematopoietic colony forming cells in bone marrow and blood, including unipotent and pluripotent progenitors. The number of CD34+ cells is greater in bone marrow than peripheral blood. Circulating bone marrow-derived immature cells like CD34+ cells have also been implicated in homeostasis of the cerebral microvasculature. Decreased levels of circulating CD34+ cells correlate with poor clinical outcomes in patients with cerebrovascular disease. Clinical trials with local transplantation of bone marrow-derived immature cells for such patients have been shown to improve impaired microcirculation [10].

The cumulative effect of two doses of autologous bone marrow derived mononuclear cells in this patient of progressive vascular dementia revealed cognitive improvement as noted on functional and neuropsychological assessment. Further, the changes on PET scan are an evidence of the clinical changes seen [15]. Hypometabolic areas such as the parietal lobes, frontal lobes, thalami, basal ganglia and temporal lobe are seen to have reached a near normal metabolic state. This normalization of metabolism leads to speculation regarding the role of the stem cells in achieving balance in the brain functioning, possibly through the above mechanisms. 
Citation: Sharma A, Badhe P, Gokulchandran N, Kulkarni P, Sane H, et al. (2012) Autologous Bone Marrow Derived Mononuclear Cell Therapy for Vascular Dementia. J Stem Cell Res Ther 2:129. doi:10.4172/2157-7633.1000129

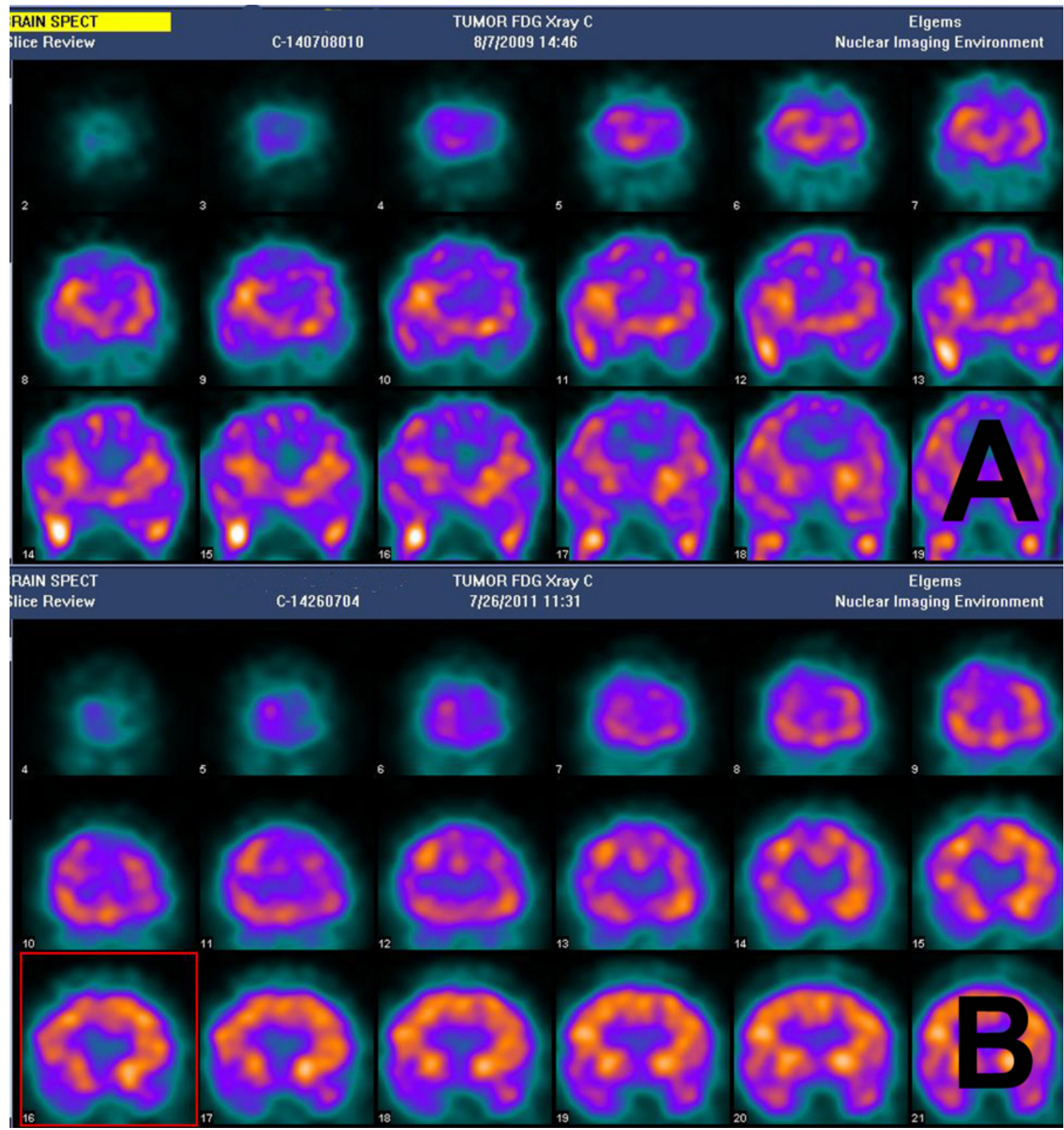

Figure 2: A) The PET scan images before the transplantation, showing white spots in the temporal lobe indicated by the arrow suggests increased FDG uptake in the temporal lobe. B) The PET scan images after 2 years, showing decrease in the FDG uptake.

Though this is just a solitary case and more evidence, in terms of numbers, needs to be gathered, it does point towards the feasibility and possible potential of the use of autologous mononuclear cell transplantation for stalling the progression of dementia.

\section{References}

1. Hagnell O, Franck A, Gräsbeck A, Ohman R, Ojesjo L, et al (1992) Vascular dementia in the Lundby study. 1. A prospective, epidemiological study of incidence and risk from 1957 to 1972. Neuropsychobiology 26: 43-49.

2. Levine DA, Langa KM (2011) Vascular cognitive impairment: disease mechanisms and therapeutic implications. Neurotherapeutics 8: 361-373.

3. Carlson RV, Boyd KM, Webb DJ (2004) The revision of the Declaration of Helsinki: past, present and future. Br J Clin Pharmacol 57: 695-713.
4. Jorm AF (1991) Cross-national comparisons of the occurrence of Alzheimer's and vascular dementias. Eur Arch Psychiatry Clin Neurosci 240: 218-222.

5. Hébert R, Brayne C (1995) Epidemiology of vascular dementia Neuroepidemiology 14: 240-257.

6. Tomlinson BE, Henderson G (1976) Some quantitative cerebral findings in normal and demented old people, in Terry RD, Gershon S (eds): Neurobiology of Aging. Raven Press Publishers. New York.

7. Black S, Román GC, Geldmacher DS, Salloway S, Hecker J, et al. (2003) Efficacy and tolerability of donepezil in vascular dementia: positive results of a 24-week, multicenter, international, randomized, placebo-controlled clinical trial. Stroke 34: 2323-2330.

8. Orgogozo JM, Rigaud AS, Stoffler A, Möbius HJ, Forette F (2002) Efficacy and safety of memantine in patients with mild to moderate vascular dementia: a randomized, placebo-controlled trial (MMM 300). Stroke 33: 1834-1839. 
Citation: Sharma A, Badhe P, Gokulchandran N, Kulkarni P, Sane H, et al. (2012) Autologous Bone Marrow Derived Mononuclear Cell Therapy for Vascular Dementia. J Stem Cell Res Ther 2:129. doi:10.4172/2157-7633.1000129

Page 4 of 4

9. Erkinjuntti T, Kurz A, Gauthier S, Bullock R, Lilienfeld S, et al. (2002) Efficacy of galantamine in probable vascular dementia and Alzheimer's disease combined with cerebrovascular disease: a randomised trial. Lancet 359: 1283-1290.

10. Taguchi A (2011) Cell-based therapy for patients with vascular dementia. Psychogeriatrics 11: 113-115.

11. Carvalho KA, Cunha RC, Vialle EN, Osiecki R, Moreira GH, et al. (2008) Functional outcome of bone marrow stem cells (CD45(+)/CD34(-)) after cell therapy in acute spinal cord injury: in exercise training and in sedentary rats. Transplant Proc 40: 847-849.

12. Bao X, Wei J, Feng M, Lu S, Li G, et al. (2011) Transplantation of human bone marrow-derived mesenchymal stem cells promotes behavioral recovery and endogenous neurogenesis after cerebral ischemia in rats. Brain Res 1367 103-113.

13. Borlongan CV, Glover LE, Tajiri N, Kaneko Y, Freeman TB (2011) The great migration of bone marrow-derived stem cells toward the ischemic brain: therapeutic implications for stroke and other neurological disorders. Prog Neurobiol 95: 213-228

14. Huang J, Yin SJ, Chen YJ, Bian WH, Yu J, et al. (2010) Transplanted bone marrow stromal cells improve cognitive dysfunction due to aging hypoperfusion in rats. Chin Med J 123: 3620-3625.

15. Bohnen NI, Djang DS, Herholz K, Anzai Y, Minoshima S (2012) Effectiveness and Safety of 18F-FDG PET in the Evaluation of Dementia: A Review of the Recent Literature. J Nucl Med 53: 59-71. 